\title{
Constraints on the optical afterglow emission of the short/hard burst GRB 010119^
}

\author{
J. Gorosabel ${ }^{1}$, M. I. Andersen ${ }^{2}$, J. Hjorth ${ }^{3}$, H. Pedersen ${ }^{3}$, B. L. Jensen ${ }^{3}$, J. U. Fynbo ${ }^{4}$, H. J. Lehto ${ }^{5,6}$, \\ S. Katajainen ${ }^{5}$, K. Hurley ${ }^{7}$, and N. Lund ${ }^{1}$ \\ 1 Danish Space Research Institute, Juliane Maries Vej 30, 2100 Copenhagen $\varnothing$, Denmark \\ e-mail: jgu@dsri.dk; nl@dsri.dk \\ 2 Division of Astronomy, University of Oulu PO Box 3000, 90014 University of Oulu, Finland \\ e-mail: michael.andersen@oulu.fi \\ 3 Astronomical Observatory, University of Copenhagen, Juliane Maries Vej 30, 2100 Copenhagen $\varnothing$, Denmark \\ e-mail: jens@astro.ku.dk; holger@astro.ku.dk; brian_j@astro.ku.dk \\ 4 European Southern Observatory, Karl-Schwarzschild-Straße 2, 85748 Garching, Germany \\ e-mail: jfynbo@eso.org \\ 5 Tuorla Observatory, Väisäläntie 20, 21500 Piikkiö, Finland \\ e-mail: harry.lehto@astro.utu.fi; seppo.katajainen@astro.utu.fi \\ 6 Department of Physics, University of Turku, 20140, Finland \\ 7 University of California, Berkeley, Space Sciences Laboratory, Berkeley, CA 94720-7450, USA \\ e-mail: khurley@ssl. berkeley.edu
}

Received 25 September 2001 / Accepted 3 December 2001

\begin{abstract}
We report optical observations of the short/hard burst GRB 010119 error box, one of the smallest error boxes reported to date for short/hard GRBs. Limits of $R>22.3$ and $I>21.2$ are imposed by observations carried out 20.31 and 20.58 hours after the gamma-ray event, respectively. They represent the most constraining limits imposed to date on the optical emission from a short/hard gamma-ray burst afterglow.
\end{abstract}

Key words. gamma rays: bursts - methods: observational - techniques: photometric

\section{Introduction}

The bimodal distribution (Hurley 1992; Kouveliotou et al. 1993) of gamma-ray bursts (GRBs) separating them into short duration $(T<2 \mathrm{~s})$ and long duration $(T>2 \mathrm{~s})$ bursts was already known before the era of Beppo-SAX. The short bursts tend to have harder spectra than the long bursts (Kouveliotou et al. 1993; Dezalay et al. 1996). Hence, in the present paper the short and long GRBs will be hereafter named as "short/hard" and "long/soft" bursts, respectively. Several years later, an analysis of the Third BATSE Catalog indicated that, in addition to these two classes of bursts, there may exist a third, intermediate soft-spectrum class of GRBs with durations $2 \mathrm{~s}<T<5 \mathrm{~s}$ (Mukherjee et al. 1998).

Send offprint requests to: J. Gorosabel, e-mail: jgu@dsri.dk

* Based on observations made with the Nordic Optical Telescope, operated on the island of La Palma jointly by Denmark, Finland, Iceland, Norway, and Sweden, in the Spanish Observatorio del Roque de los Muchachos of the Instituto de Astrofísica de Canarias.
Although the existence of this third intermediate duration class of GRBs is still under debate, in this paper we have considered them as a separate class. Thus, we consider a tri-modal distribution of GRBs; short/hard $(T<2 \mathrm{~s})$, intermediate $(2 \mathrm{~s}<T<5 \mathrm{~s})$ and long/soft $(T>5 \mathrm{~s})$ bursts.

In the simplest scenario, the short/hard bursts may be explained by the merging of the two compact components of a binary system (Lattimer \& Schramm 1974; Eichler et al. 1989), although other more exotic theories like the evaporation of primordial black-holes could also explain the observed properties of short/hard bursts (Page \& Hawking 1976; Cline et al. 1999). According to the merging model, GRBs would occur in very-low density environments, with very faint or even no afterglows at all. The observed properties of long/soft bursts can be better accommodated in the context of the collapsar model (MacFadyen \& Woosley 1999).

Castro-Tirado et al. (2001) have recently reported the possible detection of the prompt optical flash $4 \mathrm{~min}$ after the short/hard burst GRB 000313, suggesting that short/hard GRBs only show optical emission shortly after 
the gamma-ray event, with no afterglows at all. This fact would favour the models that relate short/hard GRBs to binary mergers in low-density environments.

All the GRBs for which optical, X-ray, and radio afterglows have been discovered to date belong to the long/soft GRB class, with the exception of a couple of potential intermediate duration GRBs (GRB 000301C, Jensen et al. 2001; GRB 991014, in't Zand et al. 2000). GRB 991106 was preliminarily classified as a possible short/hard GRB (Gandolfi et al. 1999a), a more detailed analysis noted that it belongs to the long/soft or intermediate class (Gandolfi et al. 1999b). Hereafter the optical upper limits reported for GRB 991106 (Castro-Tirado et al. 1999; Williams et al. 1999; Jensen et al. 1999; Gorosabel et al. 1999) will not be considered as constraints on short/hard GRB afterglows. Thus, no afterglows have been detected to date for short/hard GRBs, so their origin as well as their distance scale remain unknown. The detection of a short/hard optical afterglow similar to the ones seen for long/soft bursts would argue against the low-density environment hypothesis.

Upper limits for the prompt optical emission (response times $<5 \mathrm{~min}, R<15$ ), as well as for the afterglow emission (response times $>5$ min, $R<16$ ) have been reported by Kehoe et al. (2001). Hurley et al. (2001a) have recently reported improved positions of four short/hard GRBs determined by the Interplanetary Network (IPN) as well as several constraining upper limits on their afterglow optical and radio emission.

Fynbo et al. (2001) have recently argued that $\sim 75 \%$ of the upper limits reported to date for long/soft GRBs are compatible with faint afterglows as the one of GRB 000630, which are unreachable with most of the current long response times and shallow detection limits. Reichart \& Yost (2001) suggest that the majority of rapidly, well-localized GRBs with undetected optical afterglows are most likely the result of extinction by dust in the circumburst medium. This idea is supported by Lazzati et al. (2000) who claim that the low detection rate can not be explained by adverse observing conditions or delay in performing the observations. Thus, the existence of intrinsically dark bursts would imply that the UV flash and the X-ray afterglow do not destroy the dust responsible of the optical extinction (Reichart 2001). Panaitescu et al. (2001) predict for short/hard GRBs faint optical afterglows, exhibiting typically $R \gtrsim 23$ a few hours after the gamma-ray event.

In the absence of optical afterglow detections from short/hard bursts, deeper and earlier upper limits on the afterglow flux is the only way to answer one of the main open questions regarding short/hard GRBs; do they exhibit optical afterglows? In the present paper we discuss this question, reporting constraining $R$ and $I$-band upper limits on the afterglow optical emission from GRB 010119. In Sect. 2 the high-energy properties as well as the localisation of GRB 010119 are reported. Section 3 describes the optical observations obtained at the Nordic Optical Telescope (NOT). In Sect. 4 our upper limits are compared

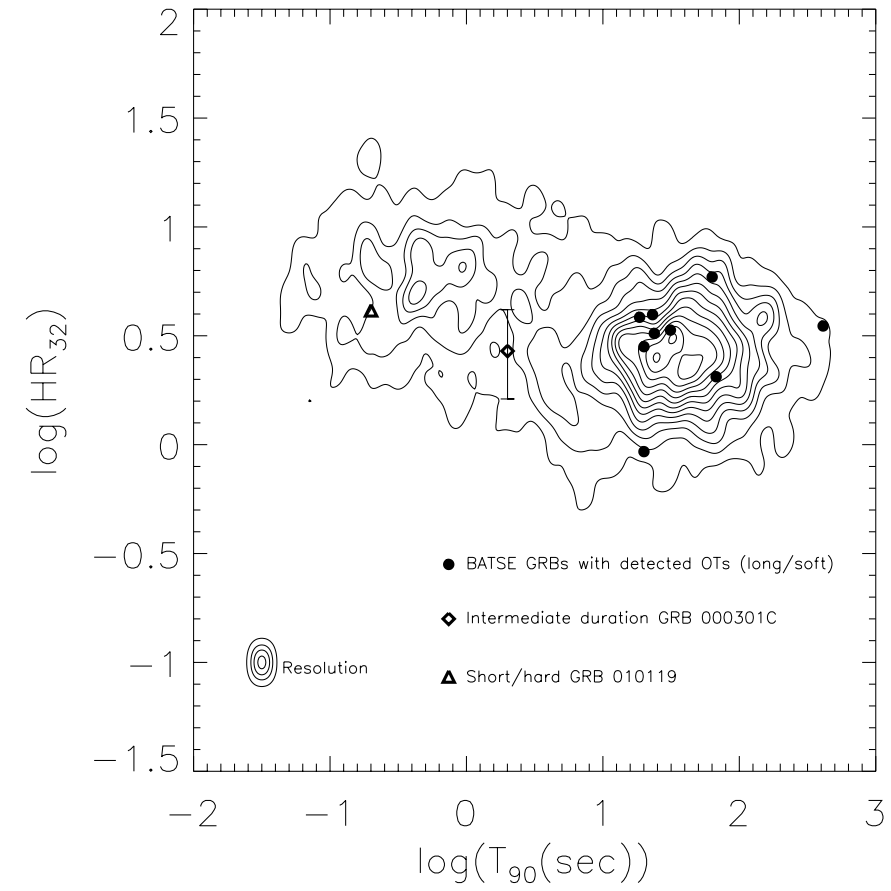

Fig. 1. A contour plot showing the duration-hardness $\left(\log \left(T_{90}\right)-\log \left(H_{32}\right)\right)$ distribution of BATSE bursts. The filled circles represent the 9 BATSE bursts with identified counterparts for which data on fluence and duration are available. All of them belong to the long/soft class of GRBs. The open diamond located in the centre of the figure represents the intermediate duration burst GRB 000301C (Jensen et al. 2001). The open triangle to the left of the plot represents GRB 010119. As can be seen, GRB 010119 belongs to the short/hard class of GRBs. Errors in the BATSE data are smaller than the symbol size. Contour levels scale linearly. The centroid in the lower left corner indicates the resolution.

to other constraints given in the literature for long/soft, intermediate, and especially short/hard GRB afterglows. Finally, Sect. 5 summarizes the conclusions of our study.

\section{Localisation of the GRB}

GRB 010119 was detected by the IPN, composed by the Ulysses, NEAR, WIND and Beppo-SAX spacecraft, on January 19.430306 UT 2001 (Hurley et al. 2001a). As observed by Ulysses, it had a duration of approximately $0.2 \mathrm{~s}$ (Hurley et al. 2001b). Integrating the analytic expression of the spectral fit of GRB 010119 given by Hurley et al. (2001a) the hardness-ratio between the BATSE channels 2 and $3\left(H_{32}\right.$; the $100-300 \mathrm{keV}$ fluence divided by the $50-100 \mathrm{keV}$ fluence) $H_{32}=4.14$ is obtained.

In Fig. 1 the duration distribution of 2115 BATSE GRBs, from the revised Fourth BATSE GRB Catalogue (Paciesas et al. 1999 and the Current BATSE GRB Catalogue at http://www.batse.msfc.nasa.gov/ batse/grb/catalog/current) is displayed. The bi-modal distribution composed by long/soft and short/hard classes of bursts can easily be seen. Additionally, we have overplotted 9 long/soft BATSE bursts with identified 
Table 1. Journal of the GRB 010119 optical observations.

\begin{tabular}{lccccccc}
\hline Telescope & Date (UT) & $\begin{array}{c}\text { Seeing } \\
\text { arcsec }\end{array}$ & Airmass & $\begin{array}{c}\text { Effective } \\
\text { Airmass }\end{array}$ & $\begin{array}{c}\text { filter } \\
(\mathrm{s})\end{array}$ & $\begin{array}{c}\text { Exp. time } \\
(3 \sigma)\end{array}$ \\
\hline NOT & $20.2703-20.2829 / 01 / 2001$ & $1.1 \times 1.8^{\star}$ & $5.34-3.84$ & 4.45 & $R$ & $3 \times 300$ & 22.3 \\
NOT & $20.2840-20.2920 / 01 / 2001$ & $1.2 \times 1.5^{\star}$ & $3.84-3.25$ & 3.57 & $I$ & $2 \times 300$ & 21.2 \\
NOT & $21.2689-21.2831 / 01 / 2001$ & $3.0^{\dagger}$ & $5.15-3.59$ & 4.27 & $R$ & $3 \times 300$ & 20.9 \\
NOT & $21.2848-21.2977 / 01 / 2001$ & $1.7^{\dagger}$ & $3.59-3.05$ & 3.36 & $I$ & $3 \times 300$ & 20.2 \\
NOT & $29.2035-29.2257 / 05 / 2001$ & 0.75 & $1.10-1.13$ & 1.11 & $R$ & $2 \times 900$ & 24.5 \\
NOT & $14.0390-14.0261 / 08 / 2001$ & 1.0 & $1.19-1.23$ & 1.21 & $I$ & $3 \times 300$ & 23.0 \\
\hline
\end{tabular}

$\star$ Elongated point spread function due to the differential chromatic refraction of the atmosphere.

$\dagger$ Through clouds.

$\dagger \dagger$ Not corrected for Galactic extinction.

afterglows for which data on fluence and duration are available (filled circles). The diamond represents the shortintermediate GRB 000301C (Jensen et al. 2001) and the triangle shows GRB 010119. As can be seen, GRB 010119 belongs to the short/hard class of gamma-ray bursts.

The coordinates of the centre of the improved GRB 010119 IPN error box are $\alpha_{2000}=18^{\mathrm{h}} 53^{\mathrm{m}} 46.17^{\mathrm{s}}$, $\delta_{2000}=11^{\circ} 59^{\prime} 47^{\prime \prime} .04$ (Hurley et al. 2001a). The size of the error box is $3.3 \mathrm{arcmin}^{2}$, significantly smaller than the preliminary $11.0 \operatorname{arcmin}^{2}$ box first reported by Hurley et al. (2001b). In fact, the error box of GRB 010119 is one of the smallest IPN error boxes reported so far for short/hard GRBs. This fact, as well as the early dissemination of the IPN position $(14.7 \mathrm{hr}$, the earliest dissemination among the 4 short/hard bursts reported by Hurley et al. 2001a), enabled us to obtain early data, providing an exceptional opportunity for detecting the counterpart of a short/hard burst.

\section{Observations}

The optical observations reported in the present paper were carried out from the NOT, equipped with the Andalucía Faint Object Spectrograph and Camera (ALFOSC). The field of view of ALFOSC $(6.5 \times 6.5)$ allowed to cover both the entire initial IPN error box, as well as the refined one. The observations started 20.16 hours after the gamma-ray event at an airmass of 5.34. The good transparency of the sky as well as the excellent seeing conditions allowed us to obtain images with a Full Width Half Maximum $(F W H M)$ star profile of $1^{\prime \prime} 1 \times 1$ 1". 8 at this extreme airmass. The stars appear elongated perpendicular to the horizon due to the atmospheric differential chromatic refraction. On May 20 and 21 (the first two nights of observations) the Sun was only $\sim 37$ degrees from the GRB field, so on these dates the observations only lasted 31.25 (May 20.2703-20.2920 UT) and $41.47 \mathrm{~min}$ (May 21.2689-21.2977) respectively, immediately before dawn (see Table 1).

Due to the high airmass and the close proximity to the Sun the background varies substantially from image to image. In order to optimize the combination of the data acquired through a given filter, the individual images have been weighted with the inverse of their mode. As the airmass gradient was very high during the exposures, it is convenient to calculate the effective airmass of the resultant co-added image. The effective airmass was calculated weighting the mean airmass of each individual exposure with the same weights used for the combination of the co-added image.

Another difficulty was the low Galactic latitude $(b=$ $4.93^{\circ}$ ) and very crowded field of GRB 010119. The corresponding Galactic extinction in the $R$ and $I$-bands are $A_{R}=1.59$ and $A_{I}=1.15 \mathrm{mag}$, respectively (Schlegel et al. 1998).

The comparison of the images was carried out with SExtractor (Bertin \& Arnouts 1996), which enabled us to deblend the overlapping stars. The co-added images were WCS calibrated and the magnitudes of the spatially coincident sources were derived. No source above the upper limits given in Table 1 exhibited magnitude differences $\Delta m>0.2$ mag in either the $R$ or $I$-bands.

The calibration was performed in August 2001 observing the Landolt field SA113 at a similar airmass as the field (Landolt 1992), which allowed us to obtain the $R$ and $I$-band magnitudes of several secondary stars in the error box. The errors in the calibration, and in the $3 \sigma$ upper limits displayed in Table 1 , are smaller than $\Delta R \lesssim 0.05 \mathrm{mag}$ and $\Delta I \lesssim 0.04 \mathrm{mag}$, respectively. These errors are of no significance for the results discussed in Sect. 4.

\section{Discussion}

We have compiled from the literature the $R$-band upper limits for long/soft $(T>5 \mathrm{~s})$, intermediate $(2 \mathrm{~s}<T<5 \mathrm{~s})$ and short/hard $(T<2 \mathrm{~s})$ GRBs for which no afterglow was found and plotted them in the left panel of Fig. 2. In order to compare our measurements only to deep observations $(R>17)$ we have not covered the region defined by the upper limits of short/hard burst afterglows given by robotic telescopes (Kehoe et al. 2001). The upper limits have been collected mainly considering Table 2 of Fynbo et al. (2001), Table 3 of Hurley et al. (2001a) and extending them by using GCN notices up to GRB 010707. In 

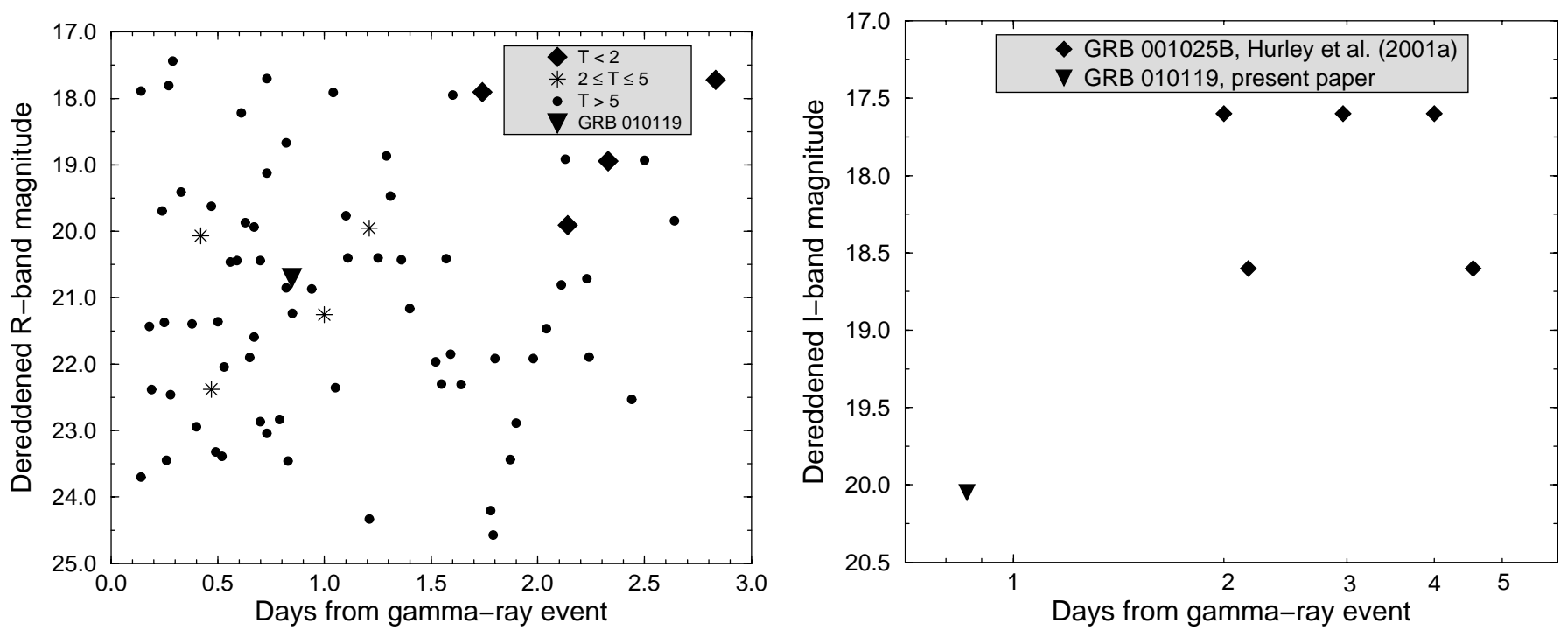

Fig. 2. Left panel: the figure shows several $R$-band upper limits for long/soft burst (circles), intermediate bursts (stars) and short/hard burst afterglows (diamonds). The upper limit imposed 20.31 hours after GRB 010119 is represented by a triangle. As can be seen, the constraint imposed by the triangle is comparable to the reported upper-limits of long/soft and intermediate GRB afterglows, and is much more constraining than the $R$-band upper limits reported to date for other short/hard GRB afterglows. Right panel: the plot displays the $I$-band upper limits reported by Hurley et al. (2001a) for GRB 001025B (diamonds) as well as the $I$-band upper limit imposed in this paper for GRB 010119, 20.58 hours after the burst (triangle). As can be seen, also in the $I$-band the triangle is more constraining than the limits reported to date for short/hard GRB afterglows. General: the measurements displayed in both panels have been dereddened following Schlegel et al. (1998).

the right panel of Fig. 2 the best $I$-band upper limits reported to date for short/hard bursts (see Table 3 of Hurley et al. 2001a) are compared to the $I$-band limit imposed for GRB 010119 in this study. All magnitudes shown if Figs. 2 and 3, as well as those discussed in the following, are corrected for their corresponding Galactic extinction (Schlegel et al. 1998).

The $R$-band upper limit presented in this paper (see triangle of the Fig. 2 left panel) is much more constraining than the other upper limits reported to date for afterglow emission from short/hard GRBs (diamonds). In fact, the $R$-band observation was done earlier and is deeper than any of the $7 R$-band upper limits previously reported by Hurley et al. (2001a).

In order to compare our $R$-band upper limits to the ones reported by other authors for short/hard GRB afterglows, all of them have to be shifted to the same epoch assuming a power law decay $F_{\nu} \sim t^{-\alpha_{R}}$ with a given value of the decay index $\alpha_{R}$. The closest diamond from the triangle in Fig. 2 left panel represents the upper limit reported by Price et al. (2001) for GRB 010119 (see Table 3 of Hurley et al. 2001a; $R>21.5 \sim 51 \mathrm{hr}$ after the GRB). This upper limit is $0.8 \mathrm{mag}$ shallower and was imposed $\sim 31$ hours later than ours (triangle). If we assume the conservative case of a shallow afterglow decay with $\alpha_{R}=1.0$, the measurement by Price et al. (2001), would correspond to a reddened magnitude of $R=20.5$ (dereddened $R=18.91$ ) at the time of our measurement. As both upper limits correspond to the same GRB, no relative Galactic reddening correction is necessary to compare the upper limits. The flux sensitivity ratio corresponding to both extincted upper limits (22.3 vs. 20.5 ) is 5.2. This relative comparison between sensitivities has to be considered as a lower limit because, in the more realistic case, $\alpha_{R}>1$ for GRB optical afterglows.

The most constraining $I$-band upper limit reported to date for a short/hard GRB afterglow corresponds to the observation carried out with the $40^{\prime \prime}$ Telescope of Las Campanas for GRB 001025B (Hurley et al. 2001a; $I>21.5,52$ hours after the GRB. See the closest diamond to the triangle of the Fig. 2 right panel). The delay and the high extinction of GRB 001025B $\left(A_{I}=2.90 \mathrm{mag}\right.$; Schlegel et al. 1998) makes this upper limit less constraining than the $I$-band observation carried out on January 20.284020.2920 UT for GRB 010119 (triangle of right panel of Fig. 2). Assuming a shallow afterglow decay of $\alpha_{I}=1.0$, we derive a contemporaneous flux sensitivity ratio of 9.6 between both upper limits, once the relative Galactic reddening factor is introduced. Therefore, the $I$-band upper limit imposed on January 20.2840-20.2920 UT ( $I>21.2$, 20.58 hours after the burst, see Table 1 ) is the most constraining upper limit imposed to date in this filter for a short/hard GRB afterglow.

From Fig. 3 it is evident that the all previous detection limits on short/hard afterglows (diamonds) would have missed most of the afterglows (only one diamond is consistent with the detectability region of the long/soft GRB afterglows, being below 3 out of 21 lightcurves, so the success ratio would be $\sim 15 \%$ ), if we make the ad-hoc assumption that short/hard bursts have afterglow characteristics similar to those of long/soft bursts. The $R$-band limit on GRB 010119 is sufficiently deep that an about $\sim 60 \%$ of 


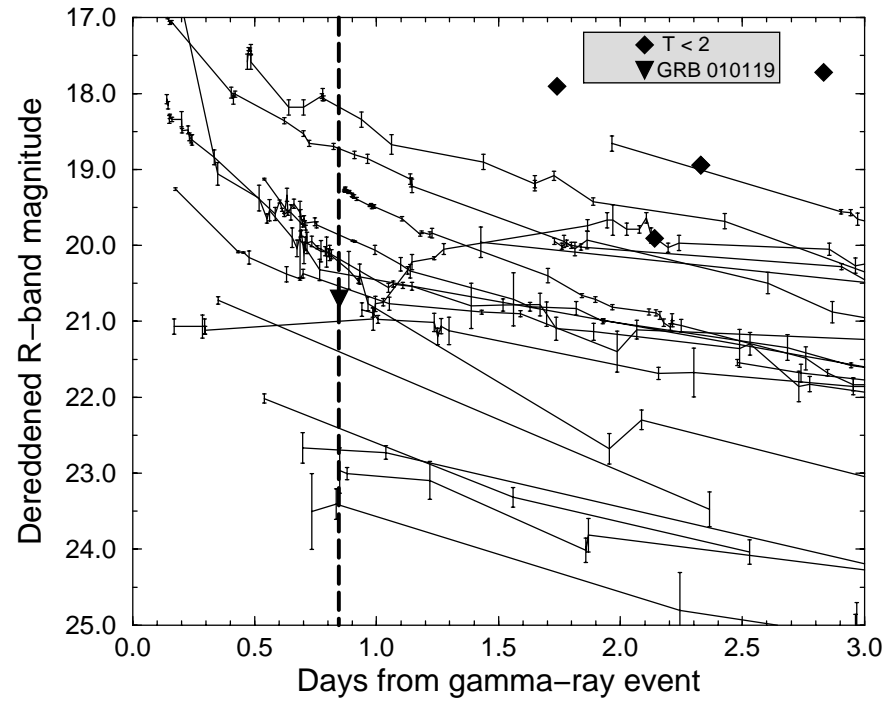

Fig. 3. The lightcurves of 22 optical afterglows for which there are detections in the $R$-band. As in the left panel of Fig. 2 the diamonds represent the $R$-band upper limits reported to date for afterglow emission from short/hard GRBs. The triangle shows the $R$-band upper limit imposed for GRB 010119. The vertical dashed band at the epoch of the GRB $010119 R$-band upper limit intersects 15 of the 22 lightcurves. Among the 15 lightcurves, 9 intersect the vertical line above the triangle. On the other hand, only one of the four diamonds (the one representing the upper limit given by Price et al. 2001) is in the detectability region, being below 3 out of 21 lightcurves (one of the 22 lightcurves does not cover the epoch of the upper limit reported by Price et al. 2001). The measurements displayed in the figure have been dereddened following Schlegel et al. (1998).

the long/soft afterglows with an $R$-band detection within a day of the burst would have been detected.

However, three out of four long bursts are never detected at optical wavelengths. Taking this into account, the afterglow detection probability for GRB 010119 would be $\sim 15 \%$. Some undetected bursts are likely dark due to late follow-up, suggesting that a more realistic detection probability with the limit achieved for GRB 010119 is of the order of $\sim 25 \%$. Independently of the fraction of intrinsically dark GRBs, the detection probability of our promptest $R$-band observation is $\sim 4$ times higher $(9 / 15$ vs. $3 / 21)$ than previous $R$-band measurements reported for short/hard GRB afterglows, assuming that short/hard and long/soft afterglows have similar characteristics.

\section{Conclusion}

The $R$-band and $I$-band limits imposed 20.31 and 20.58 hours after the gamma-ray event represent the most constraining measurements reported to date on the optical afterglow emission from a short/hard burst.

If GRB 010119 had shown an optical evolution similar to the typical long/soft and intermediate duration optical afterglows, our observations would have had a probability of $\sim 60 \%$ to detect its $R$-band counterpart. Assuming the conservative case that only $\sim 25 \%$ of the long/soft bursts exhibit optical emission, a lower limit of $\sim 15 \%$ is derived for the success ratio of our R-band upper limit.

Therefore, our observations are compatible with a completely dark short/hard GRB afterglow (as suggested by Castro-Tirado et al. 2001) or with a long/soft-like burst with faint optical emission as GRB 000630 (Fynbo et al. 2001). Our upper limits are also consistent with the predictions given by Panaitescu et al. (2001) for short/hard GRB optical afterglows.

A large number of constraining upper limits would be necessary to clarify whether short/hard bursts do not exhibit afterglows, as expected in the context of the lowdensity environments.

Acknowledgements. JG acknowledges the receipt of a Marie Curie Research Grant from the European Commission. MA acknowledges the support of the University of Oulu astrophysics group. JH acknowledges support from the Danish Natural Science Research Council (SNF). SK wishes to thank the Finnish Academy of Science and Letters (Academia Scientiarum Fennica) for support. The work of HJL is partially funded by Finnish Academy grants number 71355 and 44011. Support for the Ulysses GRB experiment is provided by JPL Contract 958056. NEAR data analysis was supported under NASA Grants NAG 5-3500 and NAG 5-9503. We also thank Scott Barthelmy for developing and maintaining the GCN, without which most counterpart searches could not be made. This research has made use of the NASA/IPC Extragalactic Database (NED) which is operated by the Jet Propulsion Laboratory, California Institute of Technology, under contract with National Aeronautics and Space Administration. We appreciate the support of the NOT staff astronomers. The data presented here have been taken using ALFOSC, which is owned by the Instituto de Astrofísica de Andalucía (IAA) and operated at the Nordic Optical Telescope under agreement between IAA and the NBIfAFG of the Astronomical Observatory of Copenhagen. We acknowledge the anonymous referee for providing helpful and constructive comments.

\section{References}

Bertin, E., \& Arnouts, S. 1996, A\&AS, 117, 393

Castro-Tirado, A. J., Gorosabel, J., Rodríguez, E., et al. 1999, GCN, 436

Castro-Tirado, A. J., Castro-Cerón, J. M., Gorosabel, J., et al. 2001, A\&A, submitted

Cline, D., Matthey, C., \& Otwinowski, S. 1999, ApJ, 527, 827

Dezalay, J.-P., Lestrade, J. P., Barat, C., et al. 1996, ApJ, 471, L27

Eichler, D., Livio, M., Piran, T., \& Schramm, D. N. 1989, Nature, 340, 126

Fynbo, J. U., Jensen, B. L., Gorosabel, J., et al. 2001, A\&A, 369,373

Gandolfi, G., Costa, E., Di Tiolo, L., et al. 1999a, GCN, 443

Gandolfi, G., Soffitta, P., Heise, J., et al. 1999b, GCN, 448

Gorosabel, J., Rol, E., Vreeswijk, P., et al. 1999, GCN, 447

Hurley, K. 1992, in Gamma-Ray Bursts, Huntsville 1991 (AIP New York) AIP Conf. Proc. 265, 3 
Hurley, K., Berger, E., Castro-Tirado, A. J., et al. 2001a, ApJ, submitted [astro-ph/0107188]

Hurley, K., Cline, T., Mazets, E., et al. 2001b, GCN, 916

Jensen, B. L., Pedersen, H., Hjorth, J., Larsen, S., \& Costa, E. 1999, GCN, 440

Jensen, B. L., Fynbo, J., Gorosabel, J., et al. 2001, A\&A, 370, 909

Kehoe, R., Akerlof, C., Balsano, R., et al. 2001, ApJ, 554, L159

Kouveliotou, C., Meegan, C. A., Fishman, G. J., et al. 1993, ApJ, 413, L101

Landolt, A. U. 1992, AJ, 104, 340

Lattimer, J. M., \& Schramm, D. N. 1974, ApJ, 192, L145

Lazzati, D., Covino, S., \& Ghisellini, G. 2000, MNRAS, submitted [astro-ph/0011443]

MacFadyen, A. I., \& Woosley, S. E. 1999, ApJ, 524, 262

Mukherjee, S., Feigelson, E. D., Babu, G. J., et al. 1998, ApJ, 508,314
Paciesas, W. S., Meegan, C. A., Pendleton, G. N., et al. 1999, ApJS, 122, 465

Page, D. N., \& Hawking, S. W. 1976, ApJ, 206, 1

Panaitescu, A., Kumar, P., \& Narayan, R. 2001, ApJ, submitted [astro-ph/0108132]

Price, P. A., Morrison, G., Bloom, J. S., et al. 2001, GCN, 919

Reichart, D. E., \& Yost, S. A. 2001, ApJ, submitted [astro-ph/0107545]

Reichart, D. E. 2001, ApJ, submitted [astro-ph/0107546]

Schlegel, D. J., Finkbeiner, D. P., \& Davis, M. 1998, ApJ, 500, 525

in't Zand, J. J. M., Kuiper, L., Amati, L., et al. 2000, ApJ, 545, 266

Williams, G. G., Park, H. S., Porrata, R., et al. 1999, GCN, 437 